UPR-0756-T, hep-th/9708023

\title{
Advances in Old-Fashioned Heterotic String Model Building
}

\author{
Gerald B. Cleaver ${ }^{\mathrm{a}}$ \\ a Department of Physics and Astronomy \\ University of Pennsylvania, Philadelphia PA 19104-6396
}

I review findings of various research groups regarding perturbative heterotic string model building in the last 12 months. Attention is given to recent studies of extra $U(1)$ 's and local discrete symmetries (LDS's) in generic string models. Issues covered include the role of $U(1)$ 's and LDS's in limiting proton decay, developments in classification of models containing anomalous $U(1)$, and possible complications resulting from kinetic mixing between observable and hidden sector $U(1)$ 's. Additionally, recent string-derived and string-inspired models are briefly reviewed.

Talk Presented at SUSY '97.

\section{Perturbative String Model Building Circa 1996-1997}

From one point of view string theory has undergone a profound paradigm shift in the last few years, a shift arguably so revolutionary that the term "string theory" will most likely be replaced by some appellation (e.g. "M"-theory or "F"theory) for the current vaguely understood underlying theory. However, from a differing viewpoint, things have not significantly changed in some aspects. The new understanding of string dualities, connecting the various string "theories" formerly thought to be independent, does not appear to significantly effect physics in the weak coupling limit of heterotic string model building (HSMB). Thus, "old fashioned" HSMB is alive and well. Weakly-coupled heterotic strings can still play an important role in model building. This talk is a review of advancements made in HSMB over the course of the last year.

Section two of the talk discusses some generic features of perturbative heterotic string models (HSMs) whose presence has no field-theoretic explanation. In particular, the role of extra local abelian and local discrete symmetries (LDS's) in HSMs are reviewed. In the (quasi-)realistic HSMs to date, such symmetries play an important role in constraining proton decay (and flavor changing neutral currents), while simultaniously allow- ing light neutrino masses [1, 7 ]. Next, progress in classification of models containing anomalous $U(1)$ are reviewed [8], including determination of anomalous $U(1)$ constraints in orbifolds. Section two concludes with discussion of HSMB complications resulting from possible kinetic mixing between observable and hidden sector $U(1)$ 's [9]. Section three briefly examines recent progress in actual HSMB.

\section{General Aspects of Model Building}

The gauge structure of the more phenomenologically realistic four-dimensional HSMs follows the general form,

$$
\begin{array}{r}
\left\{S U(3)_{c} \times S U(2)_{l} \times U(1)_{Y} \in G\right. \\
\left.\times \sum_{i} U(1)_{i} \times \sum_{j} \Delta_{j}\right\}_{\mathrm{obs}} \times \\
\left\{\tilde{G}_{\mathrm{NA}} \times \sum_{k} \tilde{U}(1)_{k} \times \sum_{l} \tilde{\Delta}_{l}\right\}_{\mathrm{hid}},
\end{array}
$$

where $\Delta_{j}$ denote LDS's. Minimally embedding the $\mathrm{SM} \mathrm{SU}(3)_{c} \times \mathrm{SU}(2)_{l}$ symmetries through free fermionic realization [12] corresponds to realizing $S U(3)_{c} \times U(1)_{c} \in S O(6)$ from charges of three complex fermions and $S U(2)_{l} \times U(1)_{l} \in S O(4)$ from those of two complex fermions. This embedding fixes the SM hypercharge to be

$U(1)_{Y}=\frac{1}{2} U(1)_{l}+\frac{1}{3} U(1)_{c}$ 


$$
=U(1)_{I_{3 R}}+\frac{1}{2} U(1)_{B-L},
$$

Non-minimal embeddings involve larger numbers of fermions and can offer degrees of freedom in the hypercharge realization. Differing hypercharge definitions can significantly alter the phenomenology of the effective field theory.

The separation between the observable and hidden sectors is generally not absolute. Indeed, most often some of the hidden sector states also carry observable $U(1)_{i}$ charges.1] Charges carried by both hidden and observable sector states are often said to belong to the "shadow sector," Although states with both shadow and hidden sector charge are generally present, quasi-realistic phenomenology requires that they have masses $m_{\text {shadow }} \geq m_{\text {susy }}$.

In the conventional supergravity (SUGRA) senario, supersymmetry (SUSY) is broken in the hidden sector at a high scale

$M_{\text {susy }}^{\text {hid }} \sim \sqrt{M_{\mathrm{Z}} M_{\mathrm{Pl}}} \sim 10^{10}$ to $12 \mathrm{GeV}$.

Gravitational interactions (and possibly Plankscale-suppressed non-renormalizable superpotential terms) then set the observable sector SUSYbreaking mass scale,

$M_{\text {susy }}^{\text {obs }} \sim\left(M_{\text {susy }}^{\text {hid }}\right)^{2} / M_{\mathrm{Pl}} \sim M_{\mathrm{Z}}$.

In the less conventional gauge-mediated process the hidden sector SUSY-breaking scale $M_{\text {susy }}^{\text {hid }}$ is typically around $100 \mathrm{TeV}$. SUSY-breaking is carried to the observable sector via loop-factor suppression factors.

Some interesting papers focusing on various roles of the ubiquitous stringy $U(1)$ 's have appeared in the last year. In the following subsections I will discuss four issues in these papers.

\section{1. $U(1)$ Beyond Those Within $\mathbf{E}_{6}$}

Recently Pati has presented evidence supporting the stringy origin of MSSM matter. Ref. [1] demonstrates that MSSM states in any phenomenologically viable model must carry at least one local $U(1)$ or LDS charge not originating from within the same $\mathrm{E}_{6}$ (or subgroup thereof)

\footnotetext{
${ }^{1}$ Furthermore, three-generation free fermionic models exist that contain exotic states carrying both observable and hidden non-Abelian charges 13.
}

(2) that $S U(3)_{c} \times S U(2)_{l} \times U(1)_{Y}$ (henceforth denoted by (321)) may be embedded into. Extra $U(1)$ 's are required for suppression of unsafe color-triplet mediated and/or gravity-linked proton decay operators generically arising in SUSY (grand)-unification. The necessity of an additional $U(1)$ becomes even more apparent when seesaw-type neutrino masses are desired. While extra $U(1)$ may be invoked ad hoc from the fieldtheoretic approach, the appearance of (several) additional $U(1)$ 's is a natural outcome in most string models.

In MSSMs, the dangerous proton decay operators arise from baryon and lepton number violating superpotential terms of the form

$$
\begin{aligned}
W & =\left[\eta_{1} U^{c} D^{c} D^{c}+\eta_{2} Q L D^{c}+\eta_{3} L L E^{c}\right] \\
& +\left[\lambda_{1} Q Q Q L+\lambda_{2} U^{c} U^{c} D^{c} E^{c}\right. \\
& \left.+\lambda_{3} L L H_{2} H_{2}\right] / M,
\end{aligned}
$$

where $\mathrm{SU}(2), \mathrm{SU}(3)$, and generational indices are suppressed. $M$ is a characteristic mass scale, assumed herein to be $M_{\text {string }} \approx g_{s} \times 5.5 \times 10^{17} \mathrm{GeV}$, where the string coupling $g_{s} \approx 0.7$.

$\eta_{i}$ and $\lambda_{j}$ represent terms of generic order and can contain built-in suppression factors $(\langle\phi\rangle / M)^{n}$, where $\langle\phi\rangle$ is a non-Abelian singlet state VEV. Proton decay limits imply $\eta_{1} \eta_{2} \lesssim$ $10^{-24}$ and $\lambda_{j} / M(\mathrm{GeV}) \lesssim 10^{-25}$ for $\Delta(B-L)=0$ decays.

While dangerous dimension four $(d=4)$ operators from renormalizable $\eta_{i}$ terms in (5) can be avoided in generic SUSY (GUT) models either by an ad hoc discrete symmetry like $R=(-1)^{3(B-L)}$ or by a local $U(1)_{B-L} \in S O(10)$ symmetry, neither symmetry can prevent similar effective $d=4$ operators from non-renormalizable $\eta_{i}$ terms containing factors of $(B-L)$ violating VEVs. Neither are the $d=5$ operators associated with the $(B-L)$-conserving, non-renormalizable $\lambda_{1}$ and $\lambda_{2}$ terms forbidden. These latter terms are expected in any theory associated with gravity unless forbidden by a new symmetry. Additionally, in SUSY GUT models, even when sufficient sup-

\footnotetext{
${ }^{2}\langle\phi\rangle$ could, in general, be replaced by a singlet product of non-Abelian fields, such as a condensate, $\langle T \bar{T}\rangle$, of two hidden sector vector-like fields. In this case, additional factors of $1 / M$ are then implied.
} 
pression of $\lambda_{j}$ is possible, induced $d=5$ operators from Higgsino triplet exchange must be prevented by doublet-triplet splitting. These operators can be avoided by fine-tuning in $\mathrm{SU}(5)$ models or by suitable choices of large numbers of and/or high-dimensionality for multiplets, as is often the method in $\mathrm{SO}(10)$ models. However, such solutions do not seem compelling, especially when stringy constraints on allowed representations (reps) are considered [3]. (See footnote 4.)

In addition, the standard seesaw mechanism assigning light masses to the (primarily) $\nu$ and heavy masses to the (primarily) $\nu^{c}$ requires breaking $(B-L)$ at a high intermediate scale. However, violating $(B-L)$ by two units still allows a discrete $R$-parity to survive, thereby eliminating the $d=4$ operators. This occurs, for example, when the VEV of a 126 of $\mathrm{SO}(10)$ gives Majorana masses to $\nu^{c}$. However, reps with dimension significantly above the adjoint's, such as $\mathrm{SO}(10)$ 's 126 , are forbidden in stringy level-one models [3] and even seem unlikely to appear at any level 跑. Alternatively, products of VEVs for sneutrino-like fields, $N^{c}$ and ${\overline{N^{\prime}}}^{c}$, from vectorlike pairs of $16_{H}, \overline{16_{H}}$ Higgs fields can play the role of the 126 and give Majorana mass to the $\nu^{c}$ from the non-renomalizable superpotential term $16_{i} \cdot 16_{i} \cdot \overline{16_{H}} \cdot \overline{16_{H}} / M$. Simultaneously these fields can produce another $\mathrm{SO}(10)$-allowed term, $16 \cdot 16 \cdot 16 \cdot 16_{H} / M$, which contains the dangerous $U^{c} D^{c} D^{c}\left\langle 16_{H}\right\rangle / M$ and $Q L D\left\langle 16_{H}\right\rangle / M$ couplings. One should expect $\left\langle 16_{H}\right\rangle / M \sim \mathcal{O}(1 / 100)$, which would predict a proton lifetime $\sim 10^{-6}$ years.

The appearance of an additional $U(1)_{\psi}^{\prime}$ from $\mathrm{E}_{6} \rightarrow \mathrm{SO}(10) \times U(1)_{\psi}^{\prime}$ improves the situation somewhat, but still doesn't eliminating fast proton decay. A 27 of $\mathrm{E}_{6}$ breaks into $\left(16_{Q_{\psi}=1}+\right.$ $\left.10_{Q_{v}=-2}+1_{Q_{\psi}=4}\right)$. The renormalizable $\eta_{i}$ terms in (5) are forbidden by $Q_{\psi}$ and $Q_{T} \equiv Q_{\psi}-(B-L)$ as well as by $(B-L)$. If required for $\nu^{c}$ masses, $\left\langle 16_{H}\right\rangle$ and $\left\langle\overline{16}_{H}\right\rangle$ break $I_{3 R},(B-L)$, and $Q_{\psi}$. $Y$ and $Q_{T}$ remain unbroken until the two MSSM Higgs in the $10_{Q_{T}=-2}$ or the singlet $1_{Q_{T}=4}$ (denoted as $\chi$ ) take on a VEV. The lowest order non-renormalizable $\eta_{i}$ terms are generated by

$$
16 \cdot 16 \cdot 16 \frac{\left\langle N^{c}\right\rangle}{M} \frac{\left\langle 10_{H}\right\rangle\left\langle 10_{H}\right\rangle}{M^{2}},
$$

$$
16 \cdot 16 \cdot 16 \frac{\left\langle N^{c}\right\rangle}{M} \frac{\langle\chi\rangle}{M},
$$

which if $\left\langle 10_{H}\right\rangle,\langle\chi\rangle \sim \mathcal{O}(1 \mathrm{TeV})$ yields $\eta_{1} \eta_{2} \ll$ $10^{-24}$.

Although the lowest order $\lambda_{j}$ terms are forbidden by both $Q_{\psi}$ and $Q_{T}$, the problem of triplet exchange exists. An effective mass term $\langle\chi\rangle H_{3} H^{\prime}{ }^{*}+$ hc for the Higgs triplets $H_{3}, H^{\prime}{ }^{\prime} \in$ $10_{H}$ breaks $Q_{\psi}$ and $Q_{T}$ by four units. Exchange of these triplets would again induce dangerous effective $d=5$ operators, with $\lambda_{j}$ above acceptable limits for $\langle\chi\rangle \sim \mathcal{O}(1 \mathrm{TeV})$.

Alternatively, Pati argues that the extraodinary stability of the proton should emerge as a compelling feature of a model, resulting from symmetries endemic to the underlying theory. The extra, ubiquitous $U(1)$ 's in heterotic string models can often form such symmetries. A typical example is the model presented in [5]. This (321) model contains a total of eight local Abelian symmeties beyond $U(1)_{I_{3 R}}$ and $U(1)_{B-L}$. The first three of these, $U(1)_{i=1,2,3}$ are generational symmetries. Specifically, all $i^{\text {th }}$ generation MSSM states carry an extra $Q_{i}=1 / 2$ charge.

Both observable and hidden sector states carry $Q_{I_{3 R}}, Q_{B-L}$, and $Q_{i=1 \text { to } 6}$ charge, while only hidden sector states have additional $Q_{i=7,8}$ charge. In this basis, $U(1)_{i=1}$ to 6 are all anomalous:

$$
\begin{aligned}
& \operatorname{Tr} U(1)_{1}=\operatorname{Tr} U(1)_{2}=\operatorname{Tr} U(1)_{3}=24 \\
& \operatorname{Tr} U(1)_{4}=\operatorname{Tr} U(1)_{5}=\operatorname{Tr} U(1)_{6}=-12 .
\end{aligned}
$$

All of the anomaly can be rotated into in a single, unique

$$
\begin{aligned}
U(1)_{\mathrm{A}}^{\prime} \equiv & c_{A} \sum_{i}\left\{\operatorname{Tr} U(1)_{i}\right\} U(1)_{i} \\
= & 2\left[U(1)_{1}+U(1)_{2}+U(1)_{3}\right] \\
& -\left[U(1)_{4}+U(1)_{5}+U(1)_{6}\right]
\end{aligned}
$$

where $c_{A}$ is a normalization coefficient. One choice of basis for the remaining non-anomalous components of the original set of $U(1)_{i}$ is

$$
\begin{aligned}
U(1)_{1}^{\prime} & =U(1)_{1}-U(1)_{2}, \\
U(1)_{2}^{\prime} & =U(1)_{4}-U(1)_{5}, \\
U(1)_{3}^{\prime} & =U(1)_{4}+U(1)_{5}-2 U(1)_{6}, \\
\hat{U}(1)_{\psi} & =U(1)_{1}+U(1)_{2}-2 U(1)_{3},
\end{aligned}
$$




$$
\begin{array}{rlr}
\hat{U}(1)_{\chi} & =\left[U(1)_{1}+U(1)_{2}+U(1)_{3}\right] \\
& +2\left[U(1)_{4}+U(1)_{5}+U(1)_{6}\right] .
\end{array}
$$

While none of the new stringy charges can singlehandedly prevent proton decay, Table 1 (borrowed from (1]) reveals the combination of either $\left(\hat{Q}_{\psi}, B-L\right)$ or $\left(\hat{Q}_{\psi}, \hat{Q}_{\chi}\right)$ can accomplish this. The charges of each pair are complimentary in their preventative roles: when one symmetry forbids a dangerous operator, the other allows it, and vice versa.

Constraints from satisfactory proton stability are minimal in this model: Either pair of symmetries need only survive from the string scale down to an intermediate scale significantly below $M_{\text {string. }}$. The sneutrino-like fields can take on VEVs up to $\sim 10^{15 \text { to } 16} \mathrm{GeV}$, and hidden sector states can condense at a scale as high as $10^{15.5} \mathrm{GeV}$. The strongest assumption is that the suppression factor, $(\langle\phi\rangle / M)^{n}$, from singlet state VEVs is $\leq 10^{-9}$.

The potential of inducing dangerous $d=5$ operators via exotic triplets in the infinite tower of Planck-mass states is entirely removed by $\hat{Q}_{\psi}$. A true symmetry in a string theory is respected by Planck-mass states as well. Thus, color-triplets in the massive tower can only appear in vectorlike pairs, with opposite $\hat{Q}_{\psi}$-charges. Such massterms cannot induce $d=5$ proton-decay operators, which violate $\hat{Q}_{\psi}$. Note that $\hat{Q}_{\psi}$ treats generations unequally, as shown in (14). This is in fact a characteristic necessary for $\hat{Q}_{\psi}$ 's success. This feature distinguishes it from $Q_{\psi}$ and implies that $\hat{Q}_{\psi}$-like symmetries cannot arise from within generation independent symmetries like $E_{6}$. There is a significant advantage of string theory over standard field theory regarding explanation of proton stability. That is, the three MSSM generations naturally arise within many classes of strings, while various string symmetries distinguish between these generations.

\subsection{Local Discrete Symmetries}

In addition to the standard proton decay operators appearing in the MSSM and the Higgsrelated in a SUSY GUT, more exotic operators can appear in non-MSSMs unless forbidden by additional symmetries. States that could gener- ate such operators inevitably appear in realistic stringy (321) models. A typical stringy example is exotic vector-like pairs of $S U(3)$ triplet/antitriplets, $\left(D^{\prime}, \bar{D}^{\prime}\right)$. Such vector pairs can produce dangerous effective $d=4$ proton-decay operators from superpotential terms of the form:

$$
\begin{aligned}
& \left\{U^{c} D^{c} \bar{D}^{\prime}, U^{c} \bar{D}^{\prime} \bar{D}^{\prime}, Q L \bar{D}^{\prime}\right. \\
& \left.U^{c} E^{c} D^{\prime}, Q Q D^{\prime}, Q h D^{\prime}\right\} \times\left(\frac{\langle\phi\rangle}{M}\right)^{n} .
\end{aligned}
$$

The first three terms in (16) are generalizations of those in (5), with $D^{c} \leftrightarrow D^{\prime}$.

However, string models often have their own means for disallowing such operators. One such method of avoidance is local discrete symmetries, $\Delta$ [6] 8]. Abelian $\Delta$ arise when a local $U(1)_{Z^{\prime}}$, with charges quantized in units of $q$, is broken by a VEV of a state with charge $N q$. Then a $Z_{N}$ LDS still survives and $\sum_{i} Q_{Z^{\prime}, i}$ in superpotential terms must equal $0(\bmod N)$. Faraggi illustrates the role of this type of symmetry in [7] $]^{3}$ In the model of [14] discussed by Faraggi, a $Z_{4}$ LDS prevents, to all orders in $n$, the appearance of all terms in (16). The $Z_{4}$ LDS remains after a local $U(1)_{Z^{\prime}}$ is broken by the VEV of a sneutrino carrying $Q_{Z^{\prime}}=\frac{1}{2}$ charge. In [14] the $Q_{Z^{\prime}}$ charges for all MSSM states are quantized in units of $n / 2$, while charges for exotic triplets are quantized in units of $n^{\prime} / 4$, with $n, n^{\prime} \in Z$. As a result, all of the potentially dangerous exotic terms carry $Q_{Z^{\prime}}$ quantized in units of $(2 n+1) / 4$. Thus, they are eliminated from the superpotential by the surviving $Z_{4}$ symmetry.

\subsection{Anomalous $U(1)$ 's}

Anomalous $U(1)$ 's such as those discussed in subsection 2.1 are typically present in compactified string models. After rotation of all anomalies into a single $U(1)_{A}^{\prime}$ via (9), the Dine-SiebergWitten (DSW) mechanism [15] breaks $U(1)_{\mathrm{A}}^{\prime}$. However, this occurs at the expense of generating a Fayet-Iliopoulos (FI) D-term that must be cancelled by VEVs of states $\Phi_{\alpha}$ carrying the anoma-

\footnotetext{
${ }^{3}$ Discussion of the roles played by stringy non-Abelian LDS's will appear in 19.
} 
lous charge,

$$
\begin{aligned}
D_{\mathrm{A}} & =\sum_{\alpha} Q_{\mathrm{A}}^{\alpha}\left|\Phi_{\alpha}\right|^{2}+\frac{g^{2}}{192 \pi^{2}} \mathrm{e}^{\phi} \operatorname{Tr} Q_{\mathrm{A}} \\
& \rightarrow 0 .
\end{aligned}
$$

Additional D- and F-flatness constraints require

$$
\begin{aligned}
D_{i} & =\sum_{\alpha} Q_{\mathrm{i}}^{\alpha}\left|\Phi_{\alpha}\right|^{2}=0, \\
F_{\alpha} & =\left\langle\partial W / \partial \Phi_{\alpha}\right\rangle=0 .
\end{aligned}
$$

The presence of an anomalous $U(1)$ has profound effects on a model. It results in (1) gauge rank reduction via the induced VEVs, (2) new non-renormalizable mass terms via the induced VEVs, (3) a new source to non-universality of soft-breaking scalar masses, and (4) new contributions to the cosmological constant through the FI D-term. Additionally, many past models have used anomalous $U(1)$ 's as generation symmetries, while recently an anomalous $U(1)$ has been used to construct models with SUSY-breaking [16, 17].

Until last year, determining if a given model contained a $U(1)_{A}^{\prime}$ meant computing the charge trace for each $U(1)_{i}$. As a first step towards classification of stringy anomalous $U(1)_{A}^{\prime}$, Kobayashi and Nakano [8] find constraints on the appearance of a $U(1)_{A}^{\prime}$ in orbifold models of $\mathrm{E}^{8} \times \mathrm{E}^{8}$ class. They show that a $U(1)_{A}^{\prime}$ will be absent if there is no massless twisted state carrying both observable and hidden sector charges or if certain types of discrete symmetries are found. Moreover, a given $U(1)_{i}$ in the observable (hidden) sector is anomaly free if there is a hidden (observable) group $G$ such that all massless $G$-charged state have vanishing charge $Q_{i}$. Relatedly, no model with an unbroken $\mathrm{E}_{8}$ can have an anomalous $U(1)_{A}^{\prime}$.

Another constraint is if a model is invariant under a $\mathrm{Z}_{2}$ momentum duality $\bar{P}_{i} \rightarrow-\bar{P}_{i}$ in the compactified direction $\mathrm{X}_{i}$, then the charges of the corresponding $U(1)_{i}$ sum to zero (with the exception that if $\bar{P}_{i}$ is a spinorial momentum, then a second, simultaneous, $\bar{P}_{j} \rightarrow-\bar{P}_{j}$ is needed).

As a stepping stone for a general $U(1)_{A}^{\prime}$ classification procedure for orbifolds, Kobayashi and Nakano demonstrate that analysis of orbifolds in a Wilson-line background can essentially be reduced to analysis in the absence of a Wilson-line.
Classification of $U(1)_{A}^{\prime}$ in terms of modular invariant shifts in a model is introduced. Of the five distinct classes of shifts found, some classes are shown to lead to anomalies while others do not. Lastly, it is suggested that LDS's will generally remain after breaking of the $U(1)_{A}^{\prime}$ and that these LDS's apply additional constraints on flat directions. There may also be a relationship between the appearance of discrete $R$-symmetries and $U(1)_{A}^{\prime}$.

Classification of $U(1)_{A}^{\prime}$ in free fermionic models is being studied by G.C. and Alon Faraggi. Translation of orbifold findings into constraints on basis vectors and phase coefficients (equivalently, $k_{i j}$-matrix entries) is underway.

\subsection{Kinetic Mixing Among $U(1)_{i}$}

Recently a new and possibly very general constraint on phenomenologically viable string models appeared [9]. Supersymmetry breaking hidden sectors containing local $\tilde{U}(1)_{k}$ hidden gauge factors could destabilize the observable gauge hierarchy under certain conditions. For both SUGRAand GUT-mediated SUSY-breaking, kinetic mixing has the potential to raise the electroweak scale up to the hidden-sector SUSY-scale, $M_{\mathrm{EW}} \rightarrow$ $M_{\text {susy }}^{\text {hid }}$. This can occur when a string model contains states that simultaneously carry both observable $U(1)_{i}$ (including $U(1)_{Y}$ ) and hidden sector $\tilde{U}(1)_{k}$ charges. This occurrence is usually the case, as discussions in prior subsections should suggest.

Such a model may contain in its pure gauge part of the Lagrangian a $U(1)$ "kinetic mixing term," with mixing strength measured by the dimensionless coefficient $\chi$ :

$$
\begin{aligned}
\mathcal{L}_{\text {gauge }}= & -\frac{1}{4} F_{(i)}^{\mu \nu} F_{(i) \mu \nu}-\frac{1}{4} F_{(k)}^{\mu \nu} F_{(k) \mu \nu} \\
& +\frac{\chi}{2} F_{(i)}^{\mu \nu} F_{(k) \mu \nu}
\end{aligned}
$$

Supersymmetrization corresponds to,

$$
\begin{array}{r}
\mathcal{L}_{\text {gauge }}=\frac{1}{32} \int d^{2} \theta\left(W_{i} W_{i}+W_{k} W_{k}\right. \\
\left.-2 \chi W_{i} W_{k}\right),
\end{array}
$$

where $W_{i} \equiv \bar{D}^{2} D V_{i}$ is the chiral gauge field strength formed from the vector superfield $V_{i}$. 
Assuming that the hidden sector $\tilde{U}(1)_{k}$ breaks when a hidden sector state with charge $\tilde{Q}_{k}$ takes a VEV, the pure gauge term in the Lagrangian may be brought to canonical form by performing the shift, $V_{i}^{\mu} \rightarrow V_{i}^{\prime}{ }^{\mu}=V_{i}^{\mu}-\chi V_{k}^{\mu}$. This shift leads to new interactions between the observable and hidden sectors:

(1) observable states $\varphi$ get hidden sector charges proportional to their observable sector charges and couplings,

$\mathcal{D}_{k}^{\mu}=\partial^{\mu}+i\left(g_{k} \tilde{Q}_{k}+g_{i} \chi Q_{i}\right) V_{k}^{\mu}$,

(2) observable states couple to hidden sector gauginos $\lambda_{k}$,

$\mathcal{L}=i \sqrt{2} g_{i} Q_{i} \chi \varphi^{\dagger} \tilde{\varphi} \lambda_{k}+$ h.c.,

(3) the hidden sector $D_{k}$ term is shifted,

$$
\begin{aligned}
D_{i}^{\prime} & =-g_{i} \sum_{a} Q_{i_{a}}\left|\varphi_{a}\right|^{2}, \\
D_{k} & =-g_{k} \sum_{b} \tilde{Q}_{k_{b}}\left|\Phi_{b}\right|^{2}+\chi D_{i}^{\prime},
\end{aligned}
$$

(4) the hidden sector $D_{k}$ term makes a contribution to soft scalar masses $\left(m_{a}^{2}\right)$ by an amount,

$\left(m_{a}^{2}\right)_{K M}=g_{i} \chi Q_{i_{a}}\left\langle D_{k}\right\rangle$.

Whether or not these mixing effects are significant depends on the ratio $\eta \equiv \chi\left\langle D_{k}\right\rangle / M_{Z}^{2} \cdot \eta \gg 1$ suggests kinetic mixing could be the dominant messenger of SUSY-breaking and produce a hierarchy destabilization as $M_{\text {scalar }} \gg M_{Z}$. While $\eta \sim \mathcal{O}(1)$ does not destablize the gauge group hierarchy, it still yields significant corrections to soft scalar masses. On the other hand, if $\eta \ll 1$ then all mixing effects are ignorable.

Stability of a model against KM effects is assured if hidden sector states appear as conjugate pairs for each $\tilde{U}(1)_{k}$ involved in hidden sector SUSY-breaking. This produces a complete cancellation of kinetic mixing terms at all orders, (i.e., $\chi=0$ ). Existence of such conjugate pairs implies a certain hidden sector $\mathrm{Z}_{2}$ LDS $\tilde{\Delta}_{l}$. For generic string models lacking such conjugate states, it is estimated that even if $\chi=0$ at $M_{\text {string }}$ at tree level, one-loop effects should generate non-zero values at lower scales,

$\frac{\chi}{g_{i} g_{k}}=\frac{b_{i k}}{16 \pi^{2}} \ln \frac{M_{\text {string }}^{2}}{\mu^{2}}+\frac{1}{16 \pi^{2}} \Delta_{i k}$.
The first term on the right-hand side of eq. (28) is the massless sector contribution, where

$b_{i k}=-\operatorname{Str}_{\text {massless }} \bar{Q}_{H}^{2} Q_{i} \tilde{Q}_{k}$,

with $\bar{Q}_{H}^{2}$ the spacetime helicity operator. The second term is an effect of the infinite tower of massive string states. Typical four-dimensional string models yield

$10^{-1} \lesssim\left|\Delta_{i k}\right| \lesssim 10^{+1}$,

which corresponds to

$3 \times 10^{-4} \lesssim \frac{\chi\left(M_{Z}\right)}{g_{b}\left(M_{Z}\right)} \lesssim 3 \times 10^{-2}$.

Thus, seemingly one should expect $\eta\left(M_{Z}\right) \gg 1$ in generic string models following either SUGRA or gauge-mediated SUSY-breaking paths.

With respect to this estimated range of typical $\chi$-values, stringy three-generation (321) models (and GUT/semi-GUT generalizations) with $N=1$ SUSY appear in striking contrast to the average model. Amazingly, in generic $N=1$ (321) models, contributions to $\chi$ cancel level-bylevel, starting with the massless states. However, the authors of [9] argue that, while cancellation is exact for tree-level masses, cancellation is unstable against mass splittings from GUT-breaking, non-zero VEVs related to the DSW mechanism, low-energy SUSY-breaking, and resulting RGEflows. $\chi / g_{k}$ contributions from mass splittings resulting from these first four effects are estimated to be of $\mathcal{O}\left(10^{-3}\right), \mathcal{O}\left(10^{-8}\right.$ to $\left.10^{-5}\right), \mathcal{O}\left(10^{-8}\right)$, and $\mathcal{O}\left(10^{-15}\right)$, respectively. Much of these range of values would yield either $\eta$ in the dangerous $\gg 1$ range or in the phenomenologically interesting $\eta \sim 1$ range. Thus, avoiding potentially dangerous kinetic mixing effects in quasi-realistic heterotic string models may imply the appearance of appropriate $Z_{2}$ LDS's in such models. However, further research into kinetic mixing appears necessary before definitive statements can be made regarding the effective low energy values of $\eta$ for the (321) models.

\section{Progress in Actual Model Building}

While definitions of string model building can vary, the practice can be grouped into two primary classes: string derived models and string 
inspired models. A string derived model essentially involves a top-down approach, starting with a true string model that yields an effective (321), semi-GUT, or GUT model in the low energy limit. On the other hand, a string inspired (321), semiGUT, or GUT model follow a more bottom-up approach. It is a field theoretic model upon which certain string-like constraints are imposed. Generally these constraints include limits on the dimensions of gauge group reps and resolution of the apparent disparity between the string scale $M_{\text {string }} \approx g_{s} \times 5.5 \times 10^{17} \mathrm{GeV}$ and the MSSM unification scale $M_{\text {unif }} \equiv 2.5 \times 10^{16} \mathrm{GeV}$.

Of the several proposed solutions to the scale disparity, findings of the last few years [10] suggest stringy GUTs 11] and/or non-MSSM states between $1 \mathrm{TeV}$ and $M_{\text {unif }}$ are perhaps the only feasible ones on the list (neglecting unknown nonperturbative effects). The Non-MSSM approach often generates semi-GUT unification near $M_{\text {unif }}$ or additional gauge factors above $M_{\text {unif }}$.

The following subsections present a cursory review of developments along both the derived and inspired lines of model construction. Stringy GUTs of the last year generally fall into the derived class 18, 19, whereas semi-GUTs 21, 22, 20 and (Near/Extended-)MSSM 23,25] tend to be of the more inspired nature.

\subsection{String Derived GUT Models}

While several groups in recent years have been pursuing string-derived SUSY GUTs [26], a sticking point has been the difficulty producing three net generations in conjunction with adjoint (or higher dimension) Higgs. Success was finally realized last year by Kakushadze and Tye using $Z_{6}$ asymmetric orbifolds that include a $Z_{3}$ outerautomorphism 18]. The latter produces level-

\footnotetext{
${ }^{4}$ For massless stringy matter to appear as a rep $R$ of a gauge group, unitarity constraints require $\lambda^{R} \cdot \Psi \leq K$, where $\lambda^{R}$ is the highest weight of $R, \Psi$ is the highest root in the gauge group, and $K$ is the level of the gauge group. For example only the $1,10,16$, and $\overline{16}$ reps can appear for $S O(10)$ at level-one. Adjoint Higgs (and higher dimension reps) can only appear at $K \geq 2$ for any gauge group. Further, the mass of a state is a linear function of the quadratic Casimir of the rep, while it is an inverse function of the level- $K$. Thus, while all $\mathrm{SO}(10)$ reps up through the 210 are allowed by unitarity at level-two, only the singlet through the 54 can be massless.
}

three current algebras yielding three-generation $S U(5), S U(6), S O(10)$, and $E_{6}$ GUTs. All models in this class contain a single adjoint (and no higher dimension reps). Discovering these models is undoubtedly the most significant string GUTs advancement of the past year. Zurab Kakushadze will speak next about these models in detail.

My study of level-two and level-fourf free fermionic $S O(10)$ models is ongoing. I have found no such models with three generations yet. While general arguments indicate three generations is more difficult to produce at these levels than at level-three, a no-go theorem does not exist. One may eventually result from my search though. However, if level-four models with three generations are ever found, they may be able to contain more than one adjoint Higgs, possibly in conjunction with 54's 佃.

\subsection{String Inspired Semi-GUT Models}

Four closely related SUSY semi-GUT models were presented recently as solutions to the MSSM-vs-string unification scale disparity. Rizos et. al 20] have developed $S U(6) \times S U(2)_{l}$ and $S U(6) \times S U(2)_{r}$ models, while Despande et. al 21] and Murayama [22] start at the string scale one step closer to the MSSM, with their $S U(4)_{c} \times S U(2)_{l} \times S U(2)_{r}$ models.

The states in these models are consistent with level-one algebras. In Rizos et. al, the gauge couplings of $S U(6)$ and $S U(2)_{l(r)}$ meet at the string scale. $S U(6) \times S U(2)_{l(r)}$ breaks to $S U(4) \times$ $S U(2)_{l} \times S U(2)_{r}$ at a scale $M_{\mathrm{X}} \approx M_{\text {unif }}$ when $(15,1)$ and $(\overline{15}, 1)$ reps acquires VEVs along $S U(4)_{c} \times S U(2)_{l} \times S U(2)_{r}$ singlet directions. At a slightly lower scale $M_{\mathrm{R}} \sim 10^{14.6 \text { to } 16.3} \mathrm{GeV}$, $S U(4)_{c} \times S U(2)_{l} \times S U(2)_{r}$ breaks to $S U(3)_{c} \times$ $S U(2)_{l} \times U(1)_{Y}$ as $(4,1,2)$ and $(\overline{4}, 1,2)$ reps. acquire VEVs along the $S U(3)$ singlet direction. Below $M_{\mathrm{R}}$, the only non-MSSM states are a pair of exotic triplets with fractional electric charge. These become massive at $10^{5}$ or $6 \mathrm{GeV}$.

In the limit of $M_{\mathrm{X}} \rightarrow M_{\text {string, }}$, one of the few differences between the $S U(6) \times S U(2)_{l}$ model and the $S U(4) \times S U(2)_{l} \times S U(2)_{r}$ models is the lowering of $M_{\mathrm{R}}$ to $10^{11}$ to $12 \mathrm{GeV}$ for the latter case.

${ }^{5} S O(10)$ level-three does not have a free fermionic realization. 
Murayama claims these models are the most efficient way of resolving the scale disparity. Each exotic state in these models plays a critical role besides simply increasing the unification scale to near $M_{\text {string }}$.

\subsection{String Inspired Extended-MSSMs}

Allanach et. al 23] have developed a class of extended MSSM models 24] that raises the MSSM coupling unification scale to approximately $M_{\text {string }}$ by the addition of extra vectorlike exotic MSSM states. Based on typical stringy anomaly cancellation requirements, the mass scale of the exotics, $M_{\mathrm{I}}$, is assumed comparable to $M_{\text {unif }}$. The exotics act on the MSSM running couplings like an RGE lens located at $M_{\mathrm{I}}$. This lens refocuses the gauge coupling unification point to a value $M_{\mathrm{X}}$ near $M_{\text {string. }}$. In these models, the (321) symmetry is enhanced above $M_{\text {I }}$ by an extra $U(1)_{X}$ generational symmetry, whose coupling merges with the others at $M_{X}$.

The range of MSSM vector-like exotics considered is limited to those permitted by level-one algebras. These are $\left[(3,2)_{Y, X},(\overline{3}, 2)_{-Y,-X}\right]$ quarklike pairs, $\left[(3,1)_{Y, X},(\overline{3}, 1)_{-Y,-X}\right]$ anti-quark-like pairs, $\left[(1,2)_{Y, X},(1,2)_{-Y,-X}\right]$ lepton doublet/ Higgs-like pairs, and $\left[(1,1)_{Y, X},(1,1)_{-Y,-X}\right]$ singlet pairs. Let $n_{Q}, n_{q}, n_{2}$, and $n_{1}$ represent the number of exotics in each of these classes, respectively. Several different combinations of $\left\{n_{Q}, n_{q}, n_{H}\right\}$ yield $M_{\mathrm{X}} \approx M_{\text {string, }}$, with the most general requirement being $n_{q}>n_{Q}+n_{2}$. The simplest combination giving $M_{X} \approx M_{\text {string }}$ is $n_{q}=3, n_{Q}=2, n_{2}=0$ plus one vector-like singlet, $\left(\Theta_{X=1}, \bar{\Theta}_{X=-1}\right)$, to break $U(1)_{X}$. Acceptable Yukawa mass textures result in more complicated models containing several vector-like Higgs (with a compensating increase in vector-like antiquarks).

Assuming a higher level for $S U(3)_{c}$ and $S U(2)_{l}$ offers an alternative answer for the scale disparity issue. Bachas et. al and Bastero-Gil et. al 25] show that (321) unification can be pushed up to $M_{\text {string }}$ by the addition of just $S U(3)_{c}$ and $S U(2)_{l}$ adjoint scalars with mass around $10^{14}$ $\mathrm{GeV}$. Since this mass scale is comparable to the scale at which coupling becomes non-perturbative for gauge groups $S U(3)$ or $S U(5)$, it is suggested that the masses of these adjoints could be related to hidden sector gaugino condensation.

\section{4. $\mu$-Term and Mass Hierarchy}

General classes of string inspired solutions have also been proposed for the $\mu$-term problem 27 29] and mass heirarchy [30,31,27,32, 33]. One of the primary inspired aspects of this pursuit is the general appearance and critical role played by extra $U(1)$ 's. Later this morning J.R. Espinosa will speak about related work here at Penn [27. So I leave further discussion of this topic to him.

\section{Concluding Comments}

"Old fashioned" heterotic string model building is definitely in its maturation stage. It has entered an era involving finely detailed (re-)analysis of (past) models. Understanding phenomenological patterns within model classes has received new attention, especially so regarding the roles of extra $U(1)$ 's and local discrete symmetries (LDS's) in the models. Extra local Abelian symmetries and LDS's play vital roles in preventing fast proton decay and flavor changing neutral currents. Moreover, whenever any extra $U(1)$ 's are anomalous, the phenomenology of the model is drastically altered. This has inspired recent study of conditions required for anomalous $U(1)$ 's to appear. Another avenue of model analysis has surfaced with the discovery of possible kinetic mixing between observable and hidden sector $U(1)$ 's. If this mixing is strong enough, possibly destablization of the observable sector gauge hierarchy could occur. LDS's in the hidden sector offer a means of eliminating this danger.

As a whole, string model building in the weakly coupled realm continues to play an important role in high energy theory. Even in the the context of the ongoing second string revolution, there is much to be gained from both the top-down and bottom-up stringy approaches to model building.

\section{ACKNOWLEDGMENTS}

G.C. thanks the SUSY ' 97 organizing committee, especially co-chairs Mirjam Cvetič and Paul Langacker, for the opportunity to speak at such a well organized and enjoyable conference. 


\section{REFERENCES}

1. J. Pati, Phys. Lett. B388 (1996) 532.

2. A. Font, L.E. Ibáñez, and F. Quevedo, Phys. Lett. B228 (1989) 79.

3. A. Font, L. Ibáñez, and F. Quevedo, Nucl. Phys. B345 (1990) 389; J. Ellis, J. Lopez, and D.V. Nanopoulos, Phys. Lett. B245 (1990) 375 .

4. K. Dienes, Nucl. Phys. B488 (1997) 141.

5. A. Faraggi, Phys. Lett. B278 (1992) 131.

6. L.E. Ibáñez and G. Ross, Nucl. Phys. B368 (1992) 3; G.K. Leontaris, Zeit. Phys. C53 (1992) 287.

7. A. Faraggi, Phys. Lett. B398 (1997) 88.

8. T. Kobayashi and H. Nakano, Nucl. Phys. B496 (1997) 103.

9. K. Dienes, C. Kolda, and J. March-Russell, Nucl. Phys. B492 (1997) 104.

10. K. Dienes and A. Faraggi, Phys. Rev. Lett. 75 (1995) 2646 and Nucl. Phys. B457 (1995) 409; K. Dienes, Phys. Rep. 287 (1997) 447 and refs. therein.

11. G. Cleaver, OHSTPY-HEP-T-96-013, hepth/9604183.

12. H. Kawai, D. Lewellen, and S.-H. Tye, Nucl. Phys. B288 (1987) 1; H. Kawai, D. Lewellen, J.A. Schwartz, and S.-H. Tye, Nucl. Phys. B299 (1988) 431; I. Antoniadis, C. Bachas, and C. Kounnas, Nucl. Phys. B289 (1987) 87; I. Antoniadis, and C. Bachas, Nucl. Phys. B298 (1988) 586.

13. S. Chaudhuri, G. Hockney, and J. Lykken, Nucl. Phys. B469 (1996) 357.

14. A. Faraggi, Phys. Lett. B302 (1993) 202.

15. M. Dine, N. Sieberg, and E. Witten, Nucl. Phys. B289 (1987) 585.

16. Duali and Pomarol, Phys. Rev. Lett. 77 (1996) 3728.

17. Binetruy and Dudas, Phys. Lett. B389 (1996) 503.

18. Z. Kakushadze and S.H. Tye, CLNS $97 / 1468$, HUTP-97/A016, HUB 3138 hep-ph/9705202 and refs. therein.

19. G. Cleaver, Free Fermionic Realizations of $S O(10)_{k}$ Embeddings. In preparation.

20. J. Rizos and K. Tamvakis, SISA/173/96/EP; hep-ph/9702295.
21. N.G. Deshpande, B. Dutta, and E. Keith, OITS-606; UCRHEP-T168; hep-ph/9611287.

22. A. Murayama, Int. J. Math. Phys. A12 (1997) 903; SH-97-01; hep-th/9705371.

23. B. Allanach and S. King, RAL 97-007; SHEP 9701; hep-ph/9703293.

24. S. Martin and P. Ramond, Phys. Rev. D51 (1995) 6515.

25. C. Bachas, C. Fabre, and T. Yanagida, Phys. Lett. B379 (1996) 49; M. BasteroGil and B. Brahmachari, Phys. Lett. B403 (1997) 51.

26. D. Lewellen, Nucl. Phys. B337 (1990) 61; S. Chaudhuri, G. Hockney and J. Lykken, hep-th/9405374, Nucl. Phys. B456 (1995) 89; G. Cleaver, hep-th/9409096, hep-th/9506006, hep-th/9604183; G. Aldazabal, A. Font, L. Ibanez and A. Uranga, Nucl. Phys. B452 (1995) 3 and Nucl. Phys. B465 (1996) 34; D. Finnell, Phys. Rev. D53 (1996) 5781; A. A. Maslikov, I. Naumov and G. G. Volkov, Int. J. Math. Phys. A11 (1996) 1117; J. Erler, Nucl. Phys. B475 (1996) 597.

27. M. Cvetič, D. Dermus, L. Everett, J.R. Espinosa, and P. Langacker, UPR-0737-T; IEMFT-150/97; hep-th/9703317; G. Cleaver, M. Cvetič, L. Everett, J.R. Espinosa, and P. Langacker, UPR-0750-T; IEM-FT-155/97; hep-ph/9705391.

28. E. Keith and E. Ma, UCRHEP-T-176; hepph/9704441.

29. Y. Kawamura, T. Kobayashi, and M. Watanabe, DPSU-97-5, hep-th/9705272.

30. Y. Kawamura and T. Kobayashi, DPSU-975; hep-th/9705272; Y. Kawamura, T. Kobayashi, and T. Komatsu, Phys. Lett. B400 (1997) 284; T. Kobayashi, and Z. Xing, Mod. Phys. Lett. A12 (1997) 561; Y. Kawamura, T. Kobayashi, and J. Kubo, DPSU-973 , hep-ph/9703320.

31. B. Allanach, S. King, G. Leontaris, and S. Lola, Phys. Rev. D56 (1997) 2632 and CERN-TH-97-45, hep-ph/9703361; B. Allanach and S. King, Phys. Lett. B407 (1997) 124.

32. A. Rasin, hep-ph/9705210.

33. J. Elwood, N. Irges, and P. Ramond, UFIFTHEP-97-17, hep-ph/9705270. 


\begin{tabular}{|c|c|c|c|c|c|c|c|}
\hline Operators & $\begin{array}{l}\text { Generation } \\
\text { Combinations }\end{array}$ & $Y$ & $B-L$ & $\hat{Q}_{\psi}$ & $\hat{Q}_{\chi}$ & $\hat{Q}_{\chi}+\hat{Q}_{\psi}$ & $\begin{array}{c}\text { If } \\
\text { Allowed }\end{array}$ \\
\hline \multirow[t]{2}{*}{$U^{c} D^{c} D^{c}, Q L D^{c}, L L E^{c}$} & $\begin{array}{l}\text { (a) All } \\
\text { except (b) }\end{array}$ & $\sqrt{ }$ & $\times$ & $\times$ & $\times$ & $\times$ & unsafe \\
\hline & $\begin{array}{l}\text { (b) } 3 \text { fields from } \\
3 \text { different } \\
\text { families }\end{array}$ & $\sqrt{ }$ & $\times$ & $\sqrt{ }$ & $\times$ & $\times$ & unsafe \\
\hline$\left(U^{c} D^{c} D^{c}\right.$ or $\left.Q L D^{c}\right)\left(N^{c} / M\right)$ & All & $\sqrt{ }$ & $\sqrt{ }$ & $x$ & $\sqrt{ }$ & $x$ & unsafe \\
\hline $\begin{array}{c}\left(U^{c} D^{c} D^{c} \text { or } Q L D^{c}\right)\left(N^{c} / M\right) \times \\
{\left[\left(\overline{h_{1}} / M\right)^{2} \text { or }(\phi / M)^{n}\right]}\end{array}$ & All & $\sqrt{ }$ & $\sqrt{ }$ & $\sqrt{ }$ & $x$ & $\times$ & safe \\
\hline $\begin{array}{c}\left(U^{c} D^{c} D^{c} \text { or } Q L D^{c}\right)\left(N^{c} / M\right) \times \\
\left(T_{a} \bar{T}_{b} / M^{2}\right)^{2}\end{array}$ & Some $(\dagger)$ & $\sqrt{ }$ & $\sqrt{ }$ & $\sqrt{ }$ & $\sqrt{ }$ & $\sqrt{ }$ & safe \\
\hline$Q Q Q L / M$ & All & $\sqrt{ }$ & $\sqrt{ }$ & $x$ & $\sqrt{ }$ & $\times$ & unsafe \\
\hline$(Q Q Q L / M)\left({\overline{N^{\prime}}}_{\alpha}^{c} / M\right)_{\alpha=1,2}$ & e.g. $(1,2,1,3)$ & $\sqrt{ }$ & $x$ & $\sqrt{ }$ & $x$ & $\times$ & unsafe \\
\hline$(Q Q Q L / M)\left({\overline{N^{\prime}}}_{\alpha}^{c} / M\right)\left(N_{\beta}^{c} / M\right)$ & All & $\sqrt{ }$ & $\sqrt{ }$ & $x$ & $\sqrt{ }$ & $x$ & safe(?) \\
\hline$(Q Q Q L / M)\left(T_{a} \bar{T}_{b} / M^{2}\right)^{2}$ & Some $(\dagger)$ & $\sqrt{ }$ & $\sqrt{ }$ & $\sqrt{ }$ & $\sqrt{ }$ & $\sqrt{ }$ & safe \\
\hline$U^{c} U^{c} D^{c} \bar{E} / M$ & All & $\sqrt{ }$ & $\sqrt{ }$ & $x$ & $\times$ & $(*)$ & unsafe \\
\hline$L L \overline{h_{i}} \overline{h_{i}} / M$ & All & $\sqrt{ }$ & $\times$ & $x$ & & $(*)$ & safe \\
\hline
\end{tabular}

Table 1

(Borrowed from [1].) The roles of $Y, B-L, \hat{Q}_{\psi}$, $\hat{Q}_{\chi}$ and $\hat{Q}_{\chi}+\hat{Q}_{\psi}$ in allowing $(\sqrt{ })$ or forbidding $(\times)$ the relevant $(B, L)$ violating operators. The mark $\nmid$ signifies that the corresponding operator is allowed if either two of the four MSSM fields are in generation 1 or 2 and two fields are in generation 3 , with $a=1$ and $b=3$ for the hidden sector states $T_{a}$ and $\bar{T}_{b}$; or all four fields are in generation 1 or 2 with $a=1$ and $b=2$. The mark (*) signifies that $\left(\hat{Q}_{\chi}+\hat{Q}_{\psi}\right)$ forbids $U^{c} U^{c} D^{c} E^{c} / M$ for all generation combinations except when all four fields belong to generation 3 , and that it forbids $L L \overline{h_{i}} \overline{h_{i}}$ in some generation combinations, but not in others. The Higgs doublet $\overline{h_{i}}$ carries generation charge $Q_{i}$. 